\title{
Correlative micro-Raman/EPMA analysis of the hydraulic calcium silicate cement interface with dentin
}

\author{
Xin $\mathrm{Li}^{1,2} \cdot$ Pong Pongprueksa $^{1} \cdot$ Kirsten Van Landuyt $^{1} \cdot$ Zhi Chen $^{2}$ • \\ Mariano Pedano $^{1}$ • Bart Van Meerbeek ${ }^{1}$ • Jan De Munck ${ }^{1}$
}

Received: 25 June 2015 / Accepted: 2 November 2015

(C) Springer-Verlag Berlin Heidelberg 2015

\begin{abstract}
Objectives This study aims to characterize the chemical interplay of hydraulic calcium silicate cements at dentin.

Materials and methods Class I cavities were prepared in noncarious human third molars and filled with Biodentine (Septodont) or ProRoot MTA (Dentsply). After 1-day, 1week, and 1-month Dulbecco's phosphate-buffered saline (DPBS) storage, the specimens were cross-sectioned perpendicular to the cement-dentin interface. The interfaces were evaluated using micro-Raman ( $\mu$ Raman) spectroscopy and at a higher spatial resolution using field emission gun electron probe microanalysis (Feg-SEM/EPMA).

Results $\mu$ Raman spectroscopy revealed the formation of a transition zone at the interface of both Biodentine (Septodont) and ProRoot MTA (Dentsply) with dentin, having an average thickness of, respectively, $7.5 \pm 4.2$ and $6.2 \pm 5.4 \mu \mathrm{m}$, which however was not statistically different. No difference in interfacial ultrastructure and chemistry was found using $\mu$ Raman spectroscopy between 1 day, 1 week, and 1 month DPBS-stored specimens. The observation of a transition zone at the cement-dentin interfaces contrasts with the EPMA data that revealed a sharper transition from cement to dentin. Again, no difference in interfacial ultrastructure and chemistry was found for different storage periods, with the exception of one 1 month DPBS-stored
\end{abstract}

Bart Van Meerbeek

bart.vanmeerbeek@med.kuleuven.be

1 BIOMAT, Department of Oral Health Sciences, KU Leuven (University of Leuven) \& Dentistry, University Hospitals Leuven, Kapucijnenvoer 7, blok A - box 7001, Leuven BE-3000, Belgium

2 The State Key Laboratory Breeding Base of Basic Science of Stomatology (Hubei-MOST) \& Key Laboratory of Oral Biomedicine Ministry of Education, School and Hospital of Stomatology, Wuhan University, Wuhan, PR, China specimen prepared using Biodentine (Septodont). More specifically, EPMA revealed a gap of about $10-\mu \mathrm{m}$ wide in the latter specimen that was filled up with newly formed calcium phosphate depositions.

Conclusions Up to 1 month, the interaction of hydraulic calcium silicate cements investigated did not reveal ultrastructural or chemical changes at unaffected dentin with the exception of a calcium phosphate gap-filling property.

Clinical relevance Hydraulic calcium silicate cements were found to fill gaps by calcium phosphate deposition, however, without conducting chemical changes to the adjacent dentin.

Keywords Hydraulic calcium silicate cement $\cdot$ Interfacial interaction $\cdot$ Micro-Raman spectroscopy $\cdot$ EPMA

\section{Introduction}

Hydraulic calcium silicate cement, often being referred to as mineral trioxide aggregate (MTA), has become the material of choice for various pulp-related treatments, such as direct and indirect pulp capping, pulpotomy, apexogenesis, repair of root perforation, treatment of in-/external root resorption, root-end filling and apical barrier formation for teeth with open apexes and necrotic pulps [1]. MTA primarily consists of Portland cement components, namely tricalcium and dicalcium silicate and tricalcium aluminate, to which bismuth oxide is added as radio-opacifier [2,3]. Major drawbacks of the currently available formulations are the long setting time [3,4], difficult handling characteristics [3,5], discoloration potential [1,6-11], and the rather difficult removal upon setting [1,12].

To overcome these shortcomings, new materials such as Biodentine (Septodont, Saint-Maur-des-Fosses, France) have been developed. Introduced as a tricalcium silicate dentin replacement material, Biodentine (Septodont) is claimed to 
possess comparable or even superior mechanical properties to those of MTA $[13,14]$. The powder/liquid formulation can be mixed in a standard dental capsule mixer, yielding an initial setting time of $12 \mathrm{~min}$ as claimed by the manufacturer. The powder is primarily composed of tricalcium and dicalcium silicate and small portions of calcium carbonate and iron oxide; zirconium oxide is added as radio-opacifier. The liquid contains, besides water, calcium chloride as a setting accelerator and a hydrosoluble polymer that serves as a waterreducing agent to ensure a good flowability despite the low water/solid ratio [14,15]. Apart from endodontic applications, Biodentine (Septodont) is also claimed for specific direct restorative applications, such as a permanent dentin replacement material to be capped with resin composite [16,17]. Its capability as a dentin remineralization agent, for instance, to remineralize caries-like demineralized dentin in order to avoid pulp exposure, is currently being explored $[18,19]$.

To date, only limited data on the interfacial interaction of hydraulic calcium silicate cements with dentin are available in scientific literature. Previous research using energy-dispersive $\mathrm{X}$-ray spectroscopy (EDS) with scanning electron microscopy (SEM) revealed the formation of a so-called "interfacial layer" with "tag-like structures" at the cement-dentin interface [20]. The latter interfacial structure was confirmed by electron probe microanalysis in combination with SEM (SEM/ EPMA), this using fully qualitative and quantitative wavelength-dispersive X-ray spectroscopy (WDS) [21]. The $\mathrm{Ca}$ and Si uptake by dentin was found to be more prominent for Biodentine (Septodont) than for White ProRoot MTA (Dentsply). Combined confocal laser scanning microscopy (CLSM), SEM, micro-Raman ( $\mu$ Raman) spectroscopy and two-photon auto-fluorescence disclosed a similar interfacial structure that was termed "mineral infiltration zone, as well as "tag-like extensions" within dentin tubules along the interface of Biodentine (Septodont) with dentin [22].

The aim of this study was to characterize the chemical interplay of two hydraulic calcium silicate cements (Biodentine, Septodont; ProRoot MTA, Dentsply) at sound dentin, this using $\mu$ Raman spectroscopy along with high-resolution fieldemission-gun electron probe microanalysis (Feg-SEM/EPMA). The null hypothesis tested was the hydraulic calcium silicate cements did not induce changes in the surface chemistry of dentin.

\section{Materials and methods}

\section{Specimen preparation}

Eighteen non-carious human third molars (gathered as approved by the Commission for Medical Ethics of KU Leuven under the file number S57622) were stored in $0.5 \%$ chloramine solution at $4{ }^{\circ} \mathrm{C}$ and were used within 2 months after extraction.
Each tooth was mounted in a gypsum block in order to facilitate manipulation. The occlusal crown third of the tooth was removed with a diamond saw (Isomet 1000, Buehler, Lake Bluff, IL, USA). One standard box-type class I cavity with the floor ending at mid-coronal dentin $(3 \times 1.5 \mathrm{~mm}$ wide, $0.5 \mathrm{~mm}$ deep $)$ was prepared in each tooth using a medium-grit $(107 \mu \mathrm{m})$ diamond bur (842, Komet, Lemgo, Germany) fixed in a watercooled high-speed turbine mounted in a custom-adapted MicroSpecimen Former (University of Iowa, Iowa City, IA, USA). All cavity surfaces were carefully verified for absence of enamel and/or pulp tissue using a stereo-microscope (Wild M5A, Heerbrugg, Switzerland). The cavities were next rinsed with distilled water for $10 \mathrm{~s}$ and air-dried, after which they were filled with either Biodentine (Septodont) or ProRoot MTA (Dentsply) (Table 1) using a plastic spatula (provided with Biodentine, Septodont) to condense the cement, while holding the gypsum blocks with the teeth on a vibrating table (Porex, Aachen, Germany). The teeth restored with Biodentine (Septodont) and ProRoot MTA (Dentsply) were kept humid by immersing the gypsum blocks in water, respectively, for $12 \mathrm{~min}$ and $4 \mathrm{~h}$, this according to the initial setting time of the two cements as mentioned by the respective manufacturers. All specimens were next immersed in Dulbecco's phosphatebuffered saline (DPBS; Lonza, Basel, Switzerland) without $\mathrm{Ca}$ and $\mathrm{Mg}$ at $37^{\circ} \mathrm{C}$ for 1 day, 1 week, and 1 month $(n=3$ per storage time). After storage, the three restored teeth per cement were cross-sectioned perpendicular to the cement-dentin interface by means of a water-cooled diamond saw (Isomet 1000, Buehler). Immediately after, the cement-dentin interface was metallo-graphically ground and polished with a Beta GrinderPolisher (Buehler) using successively 1200-grit SiC paper under running water, diamond slurries of 6 and $3 \mu \mathrm{m}$ (Kemet, Maidstone, UK) on a Texmet polishing pad (Buehler), and finally using a $1-\mu \mathrm{m}$ diamond slurry (Kemet) on a MasterTex polishing pad (Buehler). After a first $\mu$ Raman spectroscopy analysis session (see "Micro-Raman spectroscopy"), all

Table 1 Materials investigated and their composition

\begin{tabular}{|c|c|c|}
\hline Material & $\begin{array}{l}\text { Lot } \\
\text { number }\end{array}$ & Composition \\
\hline $\begin{array}{l}\text { Biodentine (Septodont, } \\
\text { Saint-Maur-des-Fosses, } \\
\text { France) }\end{array}$ & B06562 & $\begin{array}{l}\text { Powder: tricalcium silicate, } \\
\text { dicalcium silicate, calcium } \\
\text { carbonate and oxide, iron } \\
\text { oxide, zirconium oxide (as } \\
\text { radio-opacifier) } \\
\text { Liquid: distilled water, calcium } \\
\text { chloride, hydrosoluble } \\
\text { polymer }\end{array}$ \\
\hline $\begin{array}{l}\text { ProRoot MTA (Dentsply, } \\
\text { Johnson, TN, USA) }\end{array}$ & 12002493 & $\begin{array}{l}\text { Powder: tricalcium silicate, } \\
\text { dicalcium silicate, tricalcium } \\
\text { aluminate, bismuth oxide (as } \\
\text { radio-opacifier) } \\
\text { Liquid: distilled water }\end{array}$ \\
\hline
\end{tabular}


specimens were processed for electron microscopy (EM) by fixation in $2.5 \%$ glutaraldehyde for $24 \mathrm{~h}$, dehydration in ascending concentrations of ethanol $(25,50,75$, and $95 \%$ for $30 \mathrm{~min}$ each, and finally $100 \%$ for $1 \mathrm{~h}$ ), and immersion in hexamethyldisilazane (HMDS) for $10 \mathrm{~min}$. After air drying, all specimens were subsequently polished using an argon ion beam (IB-09010CP Cross Section Polisher, JEOL, Tokyo, Japan) at $5.0 \mathrm{kV}$ for $7 \mathrm{~h}$ to achieve an ion-beam polished interfacial area of approximately $1 \mathrm{~mm}^{2}$.

\section{Micro-Raman spectroscopy}

The cement-dentin interface of each specimen was analyzed using $\mu$ Raman spectroscopy (Senterra, Bruker, Billerica, MA, USA) at three stages: first after metallo-graphical polishing (first stage); next after fixation, ethanol dehydration, and HMDS drying (second stage); and finally after ion-beam polishing (third stage). At each stage, one $\mu$ Raman spectroscopy line scan analysis was performed perpendicular to the cement-dentin interface for a length of $290 \mu \mathrm{m}$ (30 points; each point $10 \mu \mathrm{m}$ apart) using a near-infrared $(785 \mathrm{~nm})$ laser, a $\times 100$ microscope objective and $50 \times 1000$ aperture, and an integration time of $10 \mathrm{~s}$ with three co-additions. For the Biodentine (Septodont) specimens, the laser power was set to $100 \mathrm{mV}$, while the laser power was reduced to $50 \mathrm{mV}$ for the ProRoot MTA (Dentsply) specimens, as it appeared more sensitive to laser damage following a pilot study. The CCD detector possessed a $1024 \times 256$ pixel resolution and was cooled down thermoelectrically to a temperature of $-65^{\circ} \mathrm{C}$. At the second and third stages, three additional $\mu$ Raman line scans for a length of $79 \mu \mathrm{m}$ ( 80 points; each point $1 \mu \mathrm{m}$ apart) as well as an area mapping were performed across the cementdentin interface using a $\times 100$ microscope objective, 50 aperture, and an integration time of $30 \mathrm{~s}$ with three co-additions. These additional scans were not conducted at the first stage, so to keep the specimens in a wet condition during $\mu$ Raman spectroscopy scanning. $\mu$ Raman peaks characteristic for dentin and the two cements were recorded. Post-processing of the spectra was performed using the Opus Spectroscopy Software version 7.0 (BrukerOptik, Ettlingen, Germany).

\section{Electron probe microanalysis}

A 2-nm-thick platinum-palladium (Pt-Pd) coating was applied to the ion-beam polished cement-dentin interface specimens by means of a turbomolecular-pumped coater (Q150T S, Quorum, East Sussex, UK). The chemical elemental composition of the cement-dentin interface was analyzed by three $180-\mu \mathrm{m}-$ long line scans as well as by an area mapping using Feg-SEM/ EPMA (JXA-8530F, JEOL, Tokyo, Japan) at high spatial resolution $( \pm 0.05 \mu \mathrm{m})$ for the elements $\mathrm{Ca}$ and P. X-ray profiles and element quantification were performed at $15 \mathrm{kV}$ (voltage) and $15 \mu \mathrm{A}$ (probe current) under high vacuum. No peak overlapping was detected.

\section{Statistical analysis}

The $\mu$ Raman as well as EPMA line analyses were processed for statistical analysis. Using an automated script, from each line, the length of the transition zone from solely "dentin" qualities towards solely "cement" qualities was measured in micrometer. The resultant values were statistically analyzed by two-way ANOVA to assess the effects of the cement (Biodentine, Septodont versus ProRoot MTA, Dentsply) and preparation method (metallo-graphical versus argon-ion-beam polishing). Tests were performed at a significance level of $\alpha=$ 0.05 using a software package (R3.01, R Foundation for Statistical Computing, Vienna, Austria).

\section{Results}

\section{$\mu$ Raman}

Raw representative $\mu$ Raman spectra of dentin and the two hydraulic calcium silicate cements are shown in Fig. 1. The intensity of the dentin characteristic phosphate $\left(\mathrm{PO}_{4}{ }^{3-}\right)$ peak at $960 \mathrm{~cm}^{-1}(\alpha)$ was used to quantify the degree of mineralization across the cement-dentin interface. The $1082 \mathrm{~cm}^{-1}$ peak $(\beta)$, which is associated with the presence of calcium carbonate, and the $1530 \mathrm{~cm}^{-1}$ peak $(\gamma)$, representing calcium silicate hydrate, were selected as peaks characteristic for Biodentine (Septodont). For ProRoot MTA (Dentsply), the cement characteristic peaks (marked with $\varepsilon$ ) ranged from 800 to $900 \mathrm{~cm}^{-1}$, but were however low in intensity in comparison with the other substrate/cement characteristic peaks. Hence, the $450 \mathrm{~cm}^{-1}(\delta)$ peak, representing bismuth, was used as cement characteristic peak for ProRoot MTA (Dentsply).

Representative $\mu$ Raman (second stage) maps superimposed on top of light microscopy surface photomicrographs of the cross-sectioned interface of Biodentine (Septodont) and ProRoot MTA (Dentsply) with dentin are presented in Fig. 2. At the cement-dentin interface of Biodentine (Septodont) (Fig. 2a-c), a transition layer of approximately $10 \mu \mathrm{m}$ between the solid dentin and bulk cement was disclosed. In this layer, the intensity of the $960 \mathrm{~cm}^{-1}(\alpha)$ dentin characteristic peak was reduced as compared to that in the solid dentin area, while the intensities of two cement characteristic peaks $(\beta, \gamma)$ reduced gradually towards dentin. Similar transition zones were detected for ProRoot MTA (Dentsply) (Fig. 2d, e) and this for both cements regardless of the 1 day, 1 week (shown in Fig. 2), and 1 month storage time. 


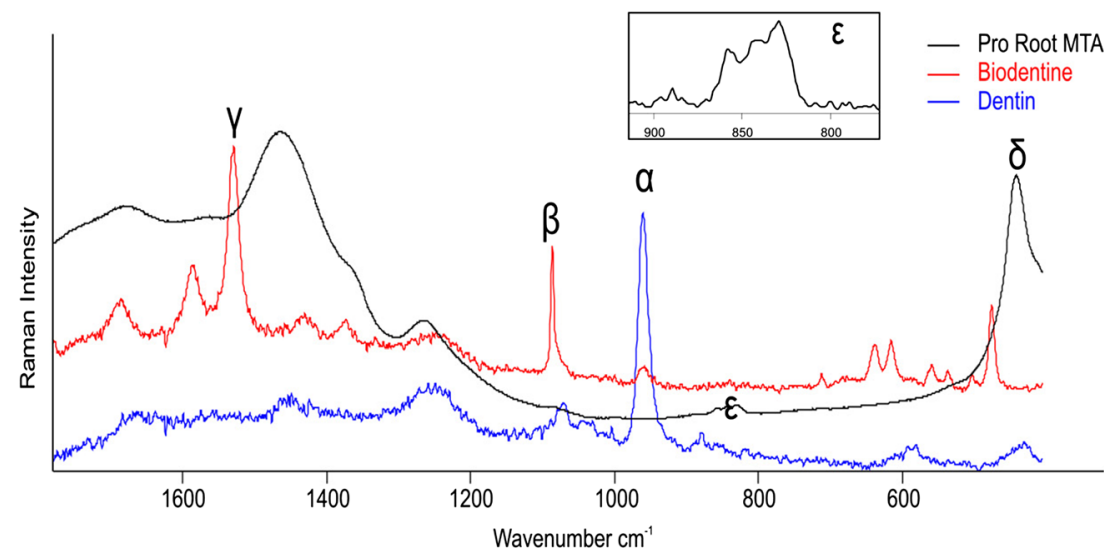

Fig. 1 Representative $\mu$ Raman spectra of dentin in blue/light grey, Biodentine (Septodont) in red/grey, and ProRoot MTA (Dentsply) in black. Characteristic peaks were selected as identifiers with the peak at $960 \mathrm{~cm}^{-1}$ (marked with $\alpha$ ) for dentin, the peaks at $1082 \mathrm{~cm}^{-1}$ (marked

with $\beta$ ), and $1530 \mathrm{~cm}^{-1}$ (marked with $\gamma$ ) for Biodentine (Septodont), and the peaks at $450 \mathrm{~cm}^{-1}$ (marked with $\delta$ ) and at the $800-900 \mathrm{~cm}^{-1}$ range (marked with $\varepsilon$; see also insert) for ProRoot MTA (Dentsply)

\section{EPMA}

Representative EPMA mapping of $\mathrm{Ca}$ across the cementdentin interface of 1 week DPBS-stored specimens and the corresponding compositional backscattered electron (BSE) photomicrographs are shown for Biodentine (Septodont) and
ProRoot MTA (Dentsply) in Fig. 3. Ca was found to have rather homogenously been spread within the bulk cement of Biodentine (Septodont) and did not quantitatively differ much from that in solid dentin (Fig. 3a). There are some Ca-free particles within the Biodentine (Septodont) cement that were identified to be composed of zirconium (star in Fig. 3a, b). As

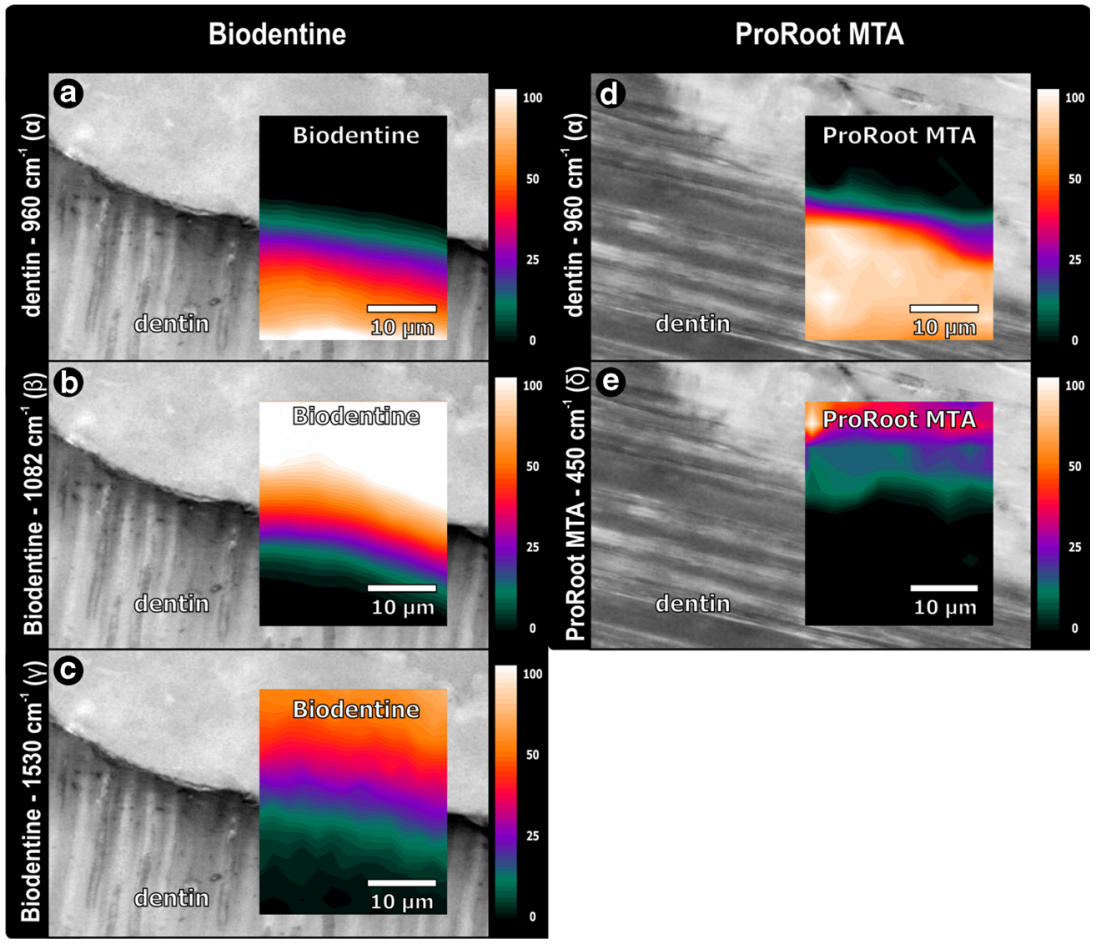

Fig. 2 Light microscopic photomicrographs and superimposed $\mu$ Raman spectroscopy mapping images across the interface of Biodentine (Septodont) with dentin for the dentin characteristic peak at $960 \mathrm{~cm}^{-1}$ ( $\alpha$ in Fig. 1) (a) and the cement-characteristic peaks at $1082 \mathrm{~cm}^{-1}(\beta$ in Fig. 1) (b) and $1530 \mathrm{~cm}^{-1}$ ( $\gamma$ in Fig. 1) (c), this upon 1-week DPBS storage of the specimen. The intensity of the dentin characteristic peak gradually decreased from dentin towards Biodentine (Septodont) with a differently colored transition zone in between (a). The intensity of the cement characteristic peaks decreased towards dentin, again disclosing an

interfacial transition zone $(\mathbf{b}, \mathbf{c})$, which were however different for the two cement characteristic peaks. Similar light microscopic photomicrographs and superimposed $\mu$ Raman spectroscopy mapping images of the cementdentin interface are shown for the dentin characteristic peak $\alpha(\mathbf{d})$ and the cement characteristic peak at $450 \mathrm{~cm}^{-1}$ ( $\delta$ in Fig. 1) (e) for ProRoot MTA (Dentsply), basically disclosing a similar transition zone as for Biodentine (Septodont). The latter cement characteristic peak $\delta$ represents bismuth oxide and varies in intensity within the cement, as being related to the random distribution of bismuth oxide particles 


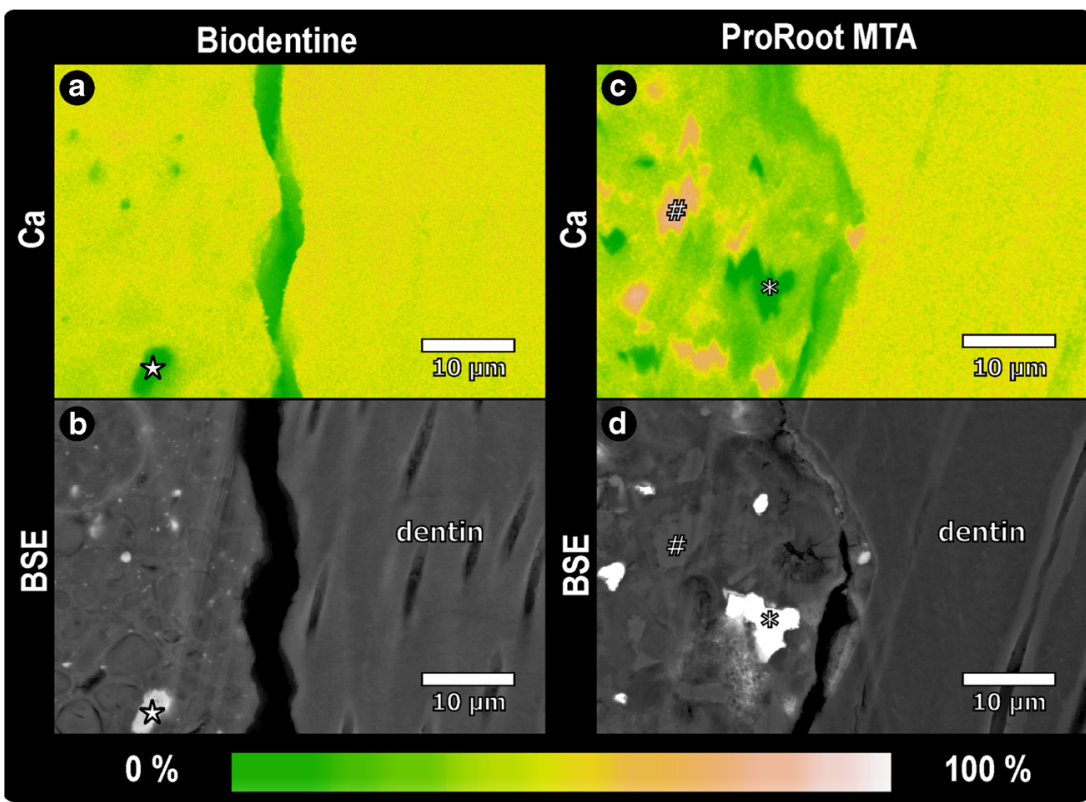

Fig. 3 EPMA chemical element mappings for Ca across the interface of Biodentine (Septodont) (a, b) and ProRoot MTA (Dentsply) (c, d) with dentin upon 1-week DPBS storage, along with the corresponding BSE images. While the Ca content of the bulk cement of Biodentine (Septodont) appeared only slightly lower than that of the solid dentin (a), that of ProRoot MTA (Dentsply) contained clearly less Ca than dentin (c). Some particles being very rich in Ca were detected in the bulk cement of ProRoot MTA (Dentsply) (\# in c), but not in that of Biodentine (Septodont) (a). Some Ca-free particles in Biodentine (Septodont) were identified as zirconium oxide particles (star in a) and correspond to the intense white particles in the corresponding BSE image (star in b). Similar particles that are low in Ca represent bismuth oxide particles in the bulk cement of ProRoot MTA (Dentsply) (asterisk in c), and again

compared to solid dentin, ProRoot MTA (Dentsply) contained overall somewhat lower $\mathrm{Ca}$, while some other cement particles contained on the other hand the highest Ca content (\# in Fig. 3c, d); other particles were composed of bismuth (asterisk in Fig. 3c, d). At the cement-dentin interface, interface debonding often occurred mostly without, but sometimes with cement remnants that remained attached to dentin. Such gap formation should in the first place be attributed to shrinkage in the high-vacuum EPMA environment. Within the dentin bulk, some variance in $\mathrm{Ca}$ concentration, mainly related to dentin tubules, can be observed, while no distinct difference in $\mathrm{Ca}$ (Fig. 3a, c) and phosphate (P; not shown) content nor ultrastructural differences were found at dentin close to the interface with Biodentine (Septodont) (Fig. 3b) and ProRoot MTA (Dentsply) (Fig. 3d).

\section{$\mu$ Raman and EPMA correlated}

Representative correlative $\mu$ Raman (third stage) and EPMA line analyses of argon-ion-beam polished cross-sectioned cement-dentin interfaces are shown in Fig. 4, each along with the corresponding light microscopy and Feg-SEM correspond to the intense white particles in the BSE image (asterisk in d). Clear interface detachment was observed for both the cements, which most likely must be ascribed to sample dehydration and the resultant shrinkage in the high-vacuum EPMA environment. In comparison with the interfacial detachment detected in Ca mapping (a), the detachment was slightly expanded in the corresponding BSE image (b) that was acquired after Ca mapping analysis. This expansion may result from the high-vacuum EPMA environment as well. While the cement de-bonded along certain parts of the interface, at other areas, cement remnants remained attached to dentin (not shown for Biodentine). No distinct zone with a change in Ca content was detected within either of the two cements or the dentin closest to the interface

photomicrographs, this for Biodentine (Septodont) (Fig. 4ad) and ProRoot MTA (Dentsply) (Fig. 4e-h) upon immersion of the specimens in DPBS for 1 week. Compared with the light microscopy image of the interface (Fig. 4a, e), the FegSEM photomicrograph (Fig. 4c, g) clearly has a higher spatial resolution and contrast. Features as individual particles, vacuum-related gaps and surface topography, which were less observable by light microscopy, were disclosed by Feg-SEM. $\mu$ Raman spectroscopy revealed that the intensity of the dentin peak decreased at the interface with the Biodentine (Septodont) cement, this over a distance of about $4 \mu \mathrm{m}$ in Fig. $4 \mathrm{~b}$ (distance between two spots $=1 \mu \mathrm{m}$ ); on average, the thickness of the transition zone, as measured by $\mu$ Raman, was $7.5 \pm 4.2 \mu \mathrm{m}$ for Biodentine (Septodont). The transition zone formed by ProRoot MTA (Dentsply) was about $6 \mu \mathrm{m}$ in Fig. $4 \mathrm{f}$ and on average $6.2 \pm 5.4 \mu \mathrm{m}$, the latter not being significantly different $(p=0.6273)$ from that measured for Biodentine (Septodont). Also, the preparation method (comparing the $\mu$ Raman data at the three specimen preparation stages) did not significantly affect the thickness of the transition area, being $5.7 \pm 4.4 \mu \mathrm{m}$ versus $8.1 \pm 5.1 \mu \mathrm{m}$ for metallographical and argon-ion-beam polishing, respectively (not shown). The cement-characteristic peaks representing 


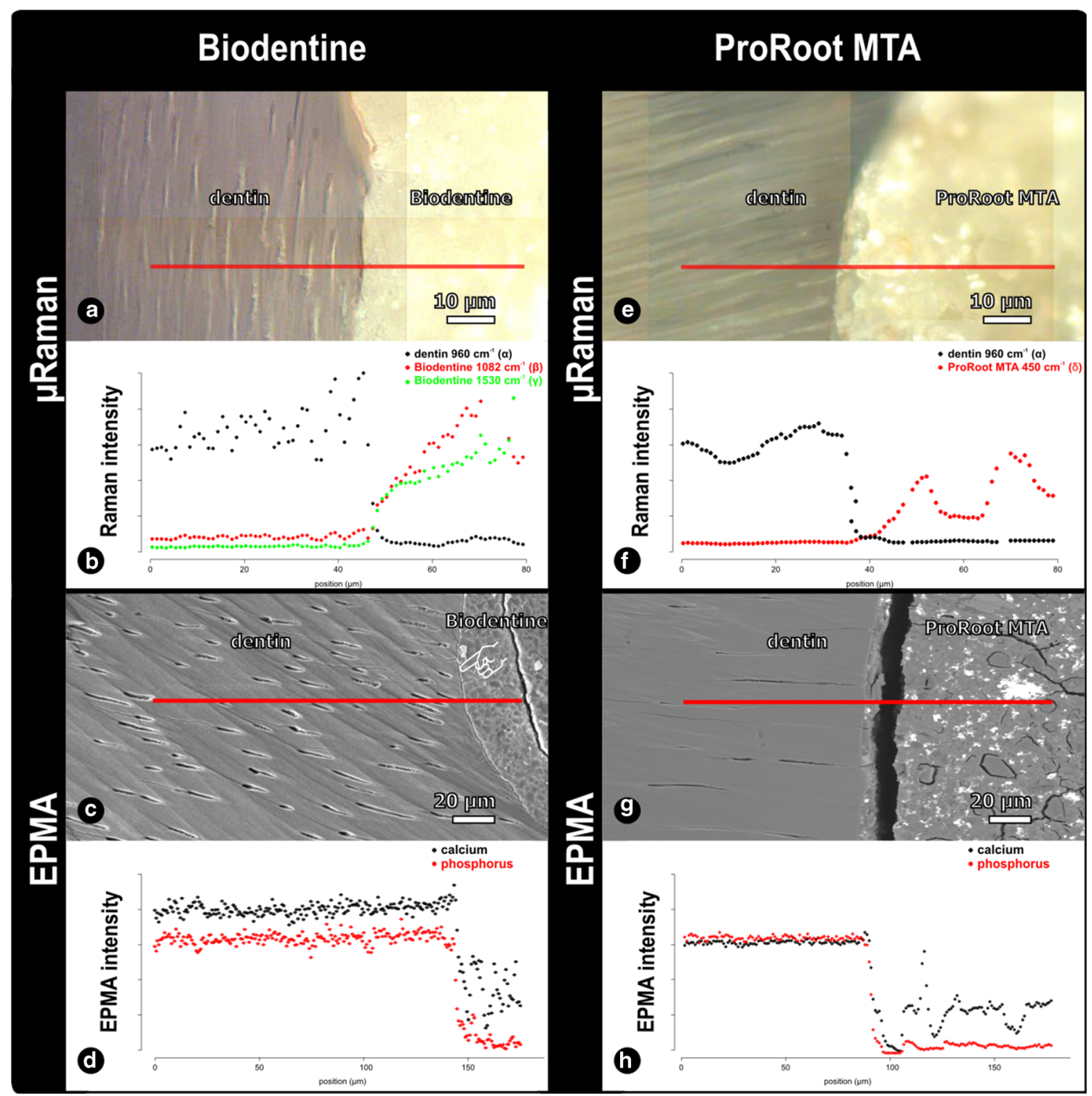

Fig. 4 Line scan chemical analysis across the interface of Biodentine (Septodont) (a-d) and ProRoot MTA (Dentsply) (e-h) with dentin upon 1 -week DPBS storage, as recorded by $\mu$ Raman spectroscopy in, respectively, $\mathbf{b}$ and $\mathbf{f}$ and by EPMA in $\mathbf{d}$ and $\mathbf{h}$. The red/black lines on the light microscopy images $(\times 100)$ that run perpendicular to the tight cementdentin interfaces (respectively, in a and e) represent the $79-\mu \mathrm{m}$ lines, along which the $\mu$ Raman data were acquired (respectively, in $\mathbf{b}$ and $\mathbf{f}$ ) for the two cements investigated. Along the cement-dentin interface of Biodentine (Septodont), the dentin-characteristic peak $\alpha$ drops in Raman intensity at the position around $46 \mu \mathrm{m}$ over a distance of about $4 \mu \mathrm{m}(\mathbf{b})$. Conversely, the cement characteristic peaks $\beta$ and $\gamma$ at, respectively, 1082 and $1530 \mathrm{~cm}^{-1}$ increase in Raman intensity at the actual interface, followed by a more gradual increase in Raman intensity towards the cement bulk (away from the interface) (b). Along the cement-dentin interface of ProRoot MTA (Dentsply), the dentin-characteristic peak $\alpha$ drops in Raman intensity at the position around $33 \mu \mathrm{m}$ over a distance of about $6 \mu \mathrm{m}$ (f). The cement characteristic peak $\delta$ at $450 \mathrm{~cm}^{-1}$ increased in Raman

Biodentine (Septodont) revealed about the same transition zone thicknesses (Fig. 4b), although more variable due to the heterogeneous material composition. Likewise, the intensity at the interface, but fluctuated further on in the cement depending of the variably distributed bismuth oxide particles within the cement (f). At a higher spatial resolution, the interfacial ultrastructures produced by Biodentine (Septodont) and ProRoot MTA (Dentsply) at dentin are more clearly depicted in the Feg-SEM photomicrographs (respectively, in $\mathbf{c}$ and $\mathbf{g}$ ). Along the cement-dentin interface of Biodentine (Septodont), a vacuum-related gap can be observed within the cement bulk (remote from the interface), while the interface of the cement with dentin appears to have remained tight (c). The changes in $\mathrm{Ca}$ and $\mathrm{P}$ content were recorded by EPMA along the $180-\mu \mathrm{m}$ long red lines that run across the respective interfaces in $\mathbf{c}$ and $\mathbf{g}$; both elements were found to drop rather abruptly in EPMA intensity at the actual interface without a clear transition zone for Biodentine (Septodont) in $\mathbf{d}$ and ProRoot MTA in $\mathbf{h}$. Along the cementdentin interface of ProRoot MTA (Dentsply), a similar vacuum-related gap with cement remnants still attached to dentin was observed (g). For both cements, $P$ remained rather low within the cement away from the interface, while the Ca content fluctuated $(\mathbf{d}, \mathbf{h})$

bismuth cement characteristic peak of ProRoot MTA (Dentsply) varied strongly in intensity within the cement bulk. 
The observation of a transition zone at the two cementdentin interfaces contrasts with the EPMA data (likewise in Fig. 3) that revealed a much more sharp transition from cement to dentin (Fig. 4d, h). This was more apparent for $\mathrm{P}$ than $\mathrm{Ca}$, with $\mathrm{P}$ not being present in Biodentine (Septodont) nor in ProRoot MTA (Dentsply) (Fig. 4d, h). Moreover, comparison of the thickness of the transition zone measured by EPMA (Fig. 4d, h) with the respective Feg-SEM photomicrographs (Fig. 4c, g) revealed that the EPMA-measured transition zone should at least in part be attributed to vacuum-related gaps at the interface.

\section{1-week versus 1-month DPBS storage}

Representative correlative Feg-SEM photomicrographs (Fig. 5a, c) and EPMA line analyses (Fig. 5b, d) of argonion-beam polished cross-sectioned cement-dentin interfaces are shown for Biodentine (Septodont) (Fig. 5a, b) and ProRoot MTA (Dentsply) (Fig. 5c, d) upon immersion of the specimens in DPBS for 1 month. The interface de-bonding observed at the interfaces of the two cements with dentin should again be related to vacuum dehydration but may also refer to a rather weak dentin-bonding potential of the two hydraulic calcium silicate cements to dentin. Similar as the 1 week DPBS-stored specimens, the 1 month DPBS-stored specimens revealed a rather sharp transition of $\mathrm{Ca}$ and $\mathrm{P}$ across the interfaces.

Comparing overall the 1-week versus the 1-month DPBS storage, no difference in either ultrastructure or interfacial chemistry was found for the two hydraulic calcium silicate cements investigated (11 specimens in total) with the exception of one 1 month specimen prepared using Biodentine (Septodont). More specifically, EPMA revealed an about 10 - $\mu \mathrm{m}$ gap that was filled up with calcium phosphate depositions (Fig. 6); they can easily be distinguished from the dentin as well as cement, as the calcium and phosphate concentrations in this newly deposited material were higher than in the respective dentin/cement bulk.

\section{Discussion}

The cement-dentin interface of two market representative hydraulic calcium silicate cements was correlatively characterized ultrastructurally using light microscopy and Feg-SEM and chemically using $\mu$ Raman and EPMA. This study aimed to explore the bioactive potential of the two cements upon interaction with dentin, when immersed in DPBS for 1 day, 1 week, and 1 month.

We correlatively used two complementary surface chemistry analyzers to detect changes in chemical composition at the

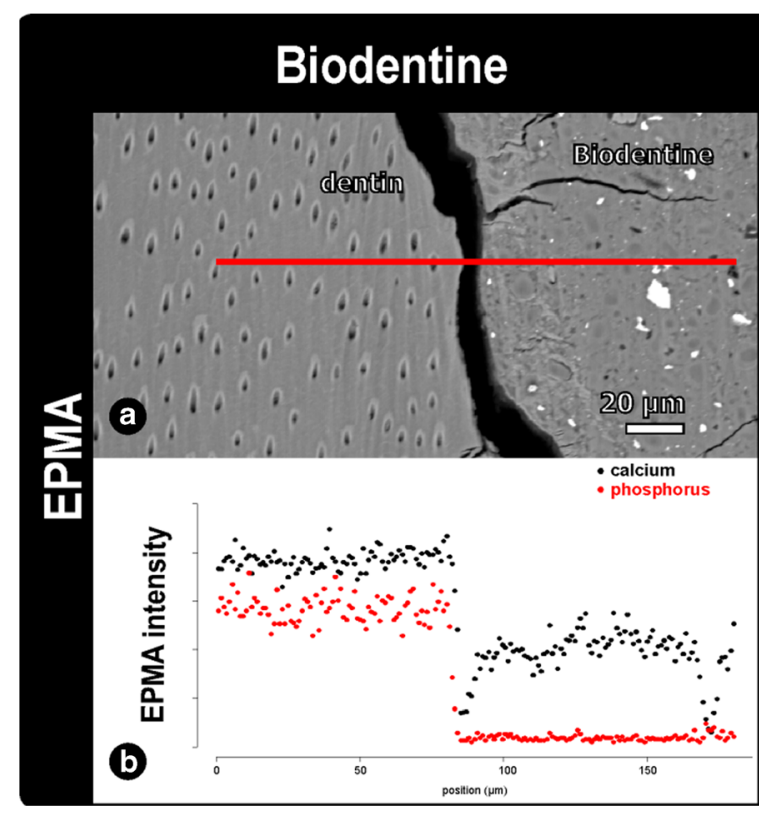

Fig. 5 Line scan chemical analysis across the interface of Biodentine (Septodont) (a, b) and ProRoot MTA (Dentsply) (c, d) with dentin upon 1 -month DPBS storage, as recorded by EPMA in $\mathbf{b}$ and $\mathbf{d}$, respectively, following the red/black lines superimposed on the corresponding FegSEM photomicrographs in a and $\mathbf{c}$, respectively. Complete interface detachment, primarily due to vacuum-related shrinkage effects, can be observed at the two cement-dentin interfaces $(\mathbf{a}, \mathbf{c})$. Both $\mathrm{Ca}$ and $\mathrm{P}$ dropped drastically in EPMA intensity at the cement-dentin interface for Biodentine (Septodont) in $\mathbf{b}$, with $\mathrm{P}$ remaining low within the cement that

\section{ProRoot MTA}

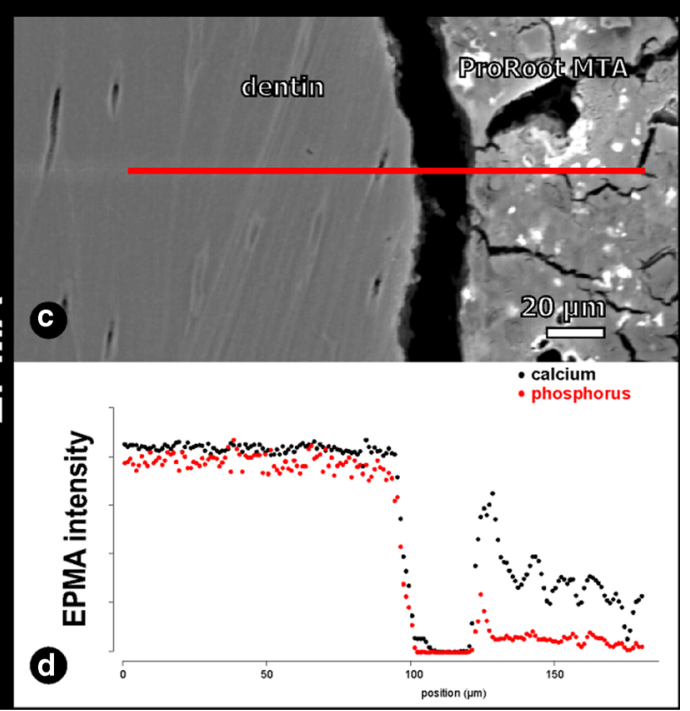

does not contain $\mathrm{P}$ and with $\mathrm{Ca}$ increasing rather gradually within the cement near the interface. Likewise, $\mathrm{Ca}$ and $\mathrm{P}$ dropped considerably in EPMA intensity at the cement-dentin interface for ProRoot MTA (Dentsply) in d, reaching zero at the interface gap. P revealed a slight and abrupt EMPA intensity peak at the outer cement layer that was attached to dentin, after which it decreased to a minimum level (d); Ca also increased rather abruptly at the outer cement layer, while it fluctuated in EPMA intensity deeper in the cement bulk (d) 
Fig. 6 Chemical EPMA mapping of the interface of Biodentine (Septodont) with dentin upon 1-month DPBS storage for $\mathrm{Ca}(\mathbf{a}), \mathrm{P}(\mathbf{b}), \mathrm{C}(\mathbf{c}), \mathrm{Si}(\mathbf{d})$, and $\mathrm{Zr}$ (e). Corresponding BSE photomicrograph (f). Clear cementdentin interface de-bonding, having resulted in an approximately $50-\mu \mathrm{m}$-wide gap, can be observed; cement fragments remained attached to dentin. Zirconium oxide particles are scattered within the cement bulk (e). Noteworthy is the layer with a higher $\mathrm{Ca}$ and $\mathrm{P}$ concentration lining the cement at the gap (hand pointer). This layer resulted also in different backscattered electron detection in $\mathbf{f}$

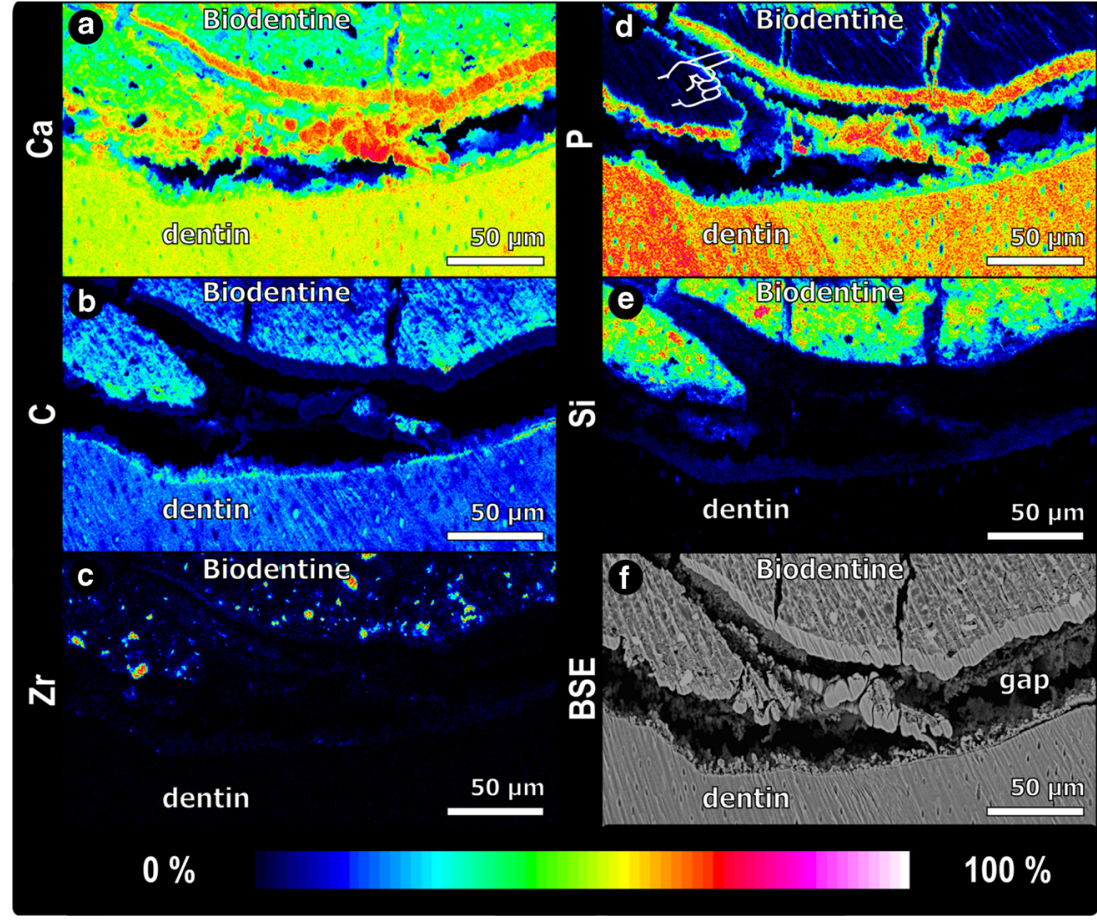

cement-dentin interface. Raman spectroscopy involves a noninvasive analytic approach for chemical identification and quantification [23]. It can probe specimens under near in vivo conditions [24,25], as well as in a solid and aqueous state $[22,23]$. Specific sample preparation is not needed, but the heat induced by the focused laser may damage the specimen's surface up to a certain depth. In this study, we needed to reduce the laser power to $50 \mathrm{mV}$ in order to avoid surface damage to the ProRoot MTA (Dentsply) specimens. EPMA is a non-destructive method, enabling full quantitative determination of elemental composition [26]. Compared with the environmental scanning electron microscope (ESEM) with an attached EDS system, which is also capable of producing element chemical analyses, EPMA requires a more extensive specimen preparation process, including dehydration and high-vacuum conditions. These may cause interfacial detachment as was observed in this study (Figs. 2, 4, and 5). However, no alterations in the interfacial element distributions are expected. Moreover, EPMA demonstrates higher spectral and lateral resolutions, which are crucial in the current research. In dental research, EPMA has been successfully used to identify changes in mineral content of both enamel and dentin [27-30]. The surface of the sample is firstly scanned with an electron beam, which excites elements to emit X-ray signals at a characteristic frequency [31]. Then, the generated element-specific X-ray signals are diffracted by analyzing crystals and counted using gas flow and sealed proportional detectors. By comparing the intensity with X-rays from standards, chemical composition is determined. $\mu$ Raman and EPMA are complementary in what they analyze (molecular versus elemental composition), regarding environment conditions (ambient versus high vacuum) and resolution (low versus high). By combining both methodologies, we were able to more profoundly (in)validate our research hypothesis advanced.

More specifically, characterization of the ultrastructure and chemistry of the cement-dentin interface using light microscopy and $\mu$ Raman, respectively, enabled the specimens to be characterized in normal room environment, basically avoiding specimen dehydration effects. Feg-SEM ultrastructural and EPMA chemical interfacial characterization required a highvacuum environment, by which specimen dehydration and shrinkage of the very dehydration-sensitive hydraulic calcium silicate cements, the wet dentin substrate, and their mutual interface resulted in vacuum-related artifact production; both the latter techniques on the other hand outperform the former techniques in particular regarding spatial resolution (see below).

Overall, with the exception of 1 out of 18 specimens, no clear changes in the surface chemistry of dentin were induced by the two cements for the three time periods; the null hypothesis was accepted. Our observation disagrees with three previous studies, which demonstrated the formation of an "interfacial layer" [20,21] or "mineral infiltration zone" [22] at the interface of a hydraulic calcium silicate cement with dentin; the latter three papers also reported the production of tag-like structures/extensions within the dentin tubules. Diverse reasons could have caused this controversy in findings.

In this study, the cement-dentin interface was additionally examined using Feg-SEM in combination with EPMA chemical analysis that has a higher spatial resolution than the 
analytic techniques employed in the three previous cementdentin interfacial characterization studies [20-22]. The resolution of $\mu$ Raman is primarily defined by the laser wavelength and microscope objective being used [32]. The typical lateral $(x-y$ direction) spatial resolution is often quoted as being in the order of $1 \mu \mathrm{m}$. Besides the laser wavelength and microscope objective, the depth $(z)$ spatial resolution depends strongly on the confocal design of the $\mu$ Raman microscope as well as the sample structure; this $z$ resolution is much more difficult to be defined. The more gradual transition of the dentin characteristic peaks towards the cement and of the cement characteristic peaks in opposite direction towards dentin, having resulted in this study in transition zones varying in width between 0.8 and $13.2 \mu \mathrm{m}$ for the two cements, should therefore be carefully interpreted; the chemical data may very likely have been captured from overlapping spots, by which a gradual transition is artificially obtained. The more abrupt transitions for $\mathrm{Ca}$ and $\mathrm{P}$ detected by Feg-SEM/EPMA confirm this assumption.

In this study, one Biodentine (Septodont) specimen revealed the deposition of calcium phosphate crystals within the produced gap (Fig. 6). This should be regarded as a gapfilling property and not be interpreted as the formation of a transition layer. Differentiation between calcium phosphatefilled gaps and the formation of a transition zone is much more difficult using lower resolution microscopy and chemical analytical tools than when Feg-SEM/EPMA is employed.

Besides difference in findings upon the use of different morphologic and chemical analytic techniques, also the specimen preparation methodology, as there are the kind of dentin the cements were applied on, the way the smear layer was produced and the way the specimens were cross-sectioned may have influenced the study outcome. In this study, the hydraulic calcium silicate cements were applied in occlusal cavities prepared in coronal dentin, as was also done in one of the previous cement-dentin interfacial characterization studies [22]. In the other two previous studies [20,21], the cements were applied into cavities of root sections, thus enabling the cements to interact with root dentin. In addition, small class I cavities were prepared using a diamond bur in this study, having resulted in the interposition of a rather compact smear layer between dentin and cement; this smear layer-covered dentin did not receive any further treatment. In the previous cement-dentin interfacial characterization studies, the smear layer was either conditioned by means of chemical reagents, namely EDTA followed by $\mathrm{NaOCl}$, as it is typically done during endodontic treatment $[20,21]$ or cleaned in an ultrasonic bath [22]. Both the surface procedures must have resulted in a dentin surface that was much less covered by smear debris than in this study. These additional smear layer treatments may have promoted cementdentin interaction, even having enabled deposition of calcium phosphate crystals within the very likely opened dentin tubules. They have been referred to as tag-like structures or extensions [20-22]. Moreover, in this study, the cements were applied in dentin cavities with the underlying root being preserved throughout the whole specimen preparation process; this is expected to have kept the dentin surface in a more natural hydrated stage. Dentin discs [22] or dentin root sections [20,21] are much more prone to dehydration but also facilitate material uptake, in particular within the tubules, by capillary forces. Another difference is that the cements have in this study been applied in small cavities rather than onto a flat surface. The cement was condensed in the cavity, while holding the tooth on a vibrating table, this to ensure proper cavity wall adaptation. Insufficient condensation and cavity wall adaptation increase the likeliness of gaps, which then may be filled with calcium phosphate depositions, as is shown in this study (Fig. 6).

A pilot study [33] revealed that commonly used metallographical polishing of cement-dentin interface cross-sections produced significant artifacts. At first, material voids can be masked, as they are plugged with smear debris or get filled up by fast dissolution and re-precipitation during polishing. Similar smearing and precipitation effects on the cross-sectioned dentin and cement surfaces also hindered qualitative/ quantitative elemental analysis. Therefore, the cement-dentin interface was in this study polished using an argon ion beam following the mechanical polishing. Using this methodology, virtually artifact-free surfaces of multi-layered structures have been reported to be obtained [34,35].

The deposition of calcium phosphate crystals in small voids, as had occurred in one specimen (Fig. 6), should be regarded as similar to the precipitation of calcium phosphate crystals onto the surface of hydraulic calcium silicate cements when they are immersed in simulated body fluid (SBF) or any other phosphate-containing fluid [36]. This process has been ascribed to the abundant release of $\mathrm{Ca}^{2+}$ ions from the set cement into the phosphate solution that is rich in $\mathrm{HPO}_{4}{ }^{2-}$ ions [37]. Upon the absorption of $\mathrm{Ca}^{2+}$ and $\mathrm{HPO}_{4}{ }^{2-}$ on the silanol groups of the silica-rich calcium silicate hydrate surface, calcium phosphate apatite is formed within the existing gaps and/or voids [37]. Using this mechanism, tricalcium silicate cements were shown to remineralize artificial caries-affected-like dentin via the deposition of minerals that occluded the dentin tubules and filled the zone of exposed collagen fibrils [18,38,39]. Accordingly, a study using MTA in conjunction with biomimetic analogs like polyacrylic acid and sodium tripolyphosphate was shown to successfully remineralize partially demineralized dentin in SBF solution [38]. Relating these data to the findings of this study, one may assume that these remineralization processes occur in affected dentin, while they might be less likely in case of unaffected dentin that is already fully mineralized.

\section{Conclusion}

At relatively short time (up to 1 month), the application and interaction of the two hydraulic calcium silicate cements 
investigated did not reveal ultrastructural or chemical changes in unaffected dentin, even at areas closest to the cement.

\section{Compliance with ethical standards}

Funding This study was funded by the Research Project grant G089315N awarded by the Research Foundation - Flanders (FWO). Drs. Xin Li's research stay at KU Leuven is supported by the China Scholarship Council (File No.201206270126).

Conflict of interest The authors declare that they have no competing interests.

Ethical approval This article does not contain any studies with human participants or animals performed by any of the authors. The human third molars employed in the study were gathered with informed consent as approved by the Commission for Medical Ethics of KU Leuven under the file number S57622.

\section{References}

1. Torabinejad M, Chivian N (1999) Clinical applications of mineral trioxide aggregate. J Endod 25:197-205

2. Camilleri J, Montesin FE, Brady K, Sweeney R, Curtis RV, Ford TR (2005) The constitution of mineral trioxide aggregate. Dent Mater 21:297-303

3. Torabinejad M, White DJ (1995) Tooth filling material and use. US Patent Number 5,769,638

4. Torabinejad M, Hong CU, McDonald F, Pitt Ford TR (1995) Physical and chemical properties of a new root-end filling material. J Endod 21:349-353

5. Budig CG, Eleazer PD (2008) In vitro comparison of the setting of dry ProRoot MTA by moisture absorbed through the root. J Endod 34:712-714. doi:10.1016/j.joen.2008.03.004

6. Vallés M, Mercadé M, Duran-Sindreu F, Bourdelande JL, Roig M (2013) Influence of light and oxygen on the color stability of five calcium silicate-based materials. J Endod 39:525-528. doi:10. 1016/j.joen.2012.12.021

7. Vallés M, Mercadé M, Duran-Sindreu F, Bourdelande JL, Roig M (2013) Color stability of white mineral trioxide aggregate. Clin Oral Investig 17:1155-1159. doi:10.1007/s00784-012-0794-1

8. Camilleri J (2014) Color stability of white mineral trioxide aggregate in contact with hypochlorite solution. J Endod 40:436-440. doi:10.1016/j.joen.2013.09.040

9. Marciano MA, Costa RM, Camilleri J, Mondelli RF, Guimarães BM, Duarte MA (2014) Assessment of color stability of white mineral trioxide aggregate angelus and bismuth oxide in contact with tooth structure. J Endod 40:1235-1240. doi:10.1016/j.joen. 2014.01.044

10. Guimarães BM, Tartari T, Marciano MA, Vivan RR, Mondeli RF, Camilleri J, Duarte MA (2015) Color stability, radiopacity, and chemical characteristics of white mineral trioxide aggregate associated with 2 different vehicles in contact with blood. J Endod 41: 947-952. doi:10.1016/j.joen.2015.02.008

11. Marciano MA, Duarte MA, Camilleri J (2015) Dental discoloration caused by bismuth oxide in MTA in the presence of sodium hypochlorite. Clin Oral Investig 30

12. Boutsioukis C, Noula G, Lambrianidis T (2008) Ex vivo study of the efficiency of two techniques for the removal of mineral trioxide aggregate used as a root canal filling material. J Endod 34:12391242. doi:10.1016/j.joen.2008.07.018

13. Natale LC, Rodrigues MC, Xavier TA, Simoes A, de Souza DN, Braga RR (2015) Ion release and mechanical properties of calcium silicate and calcium hydroxide materials used for pulp capping. Int Endod J 48:89-94. doi:10.1111/iej.12281

14. Grech L, Mallia B, Camilleri J (2013) Investigation of the physical properties of tricalcium silicate cement-based rootend filling materials. Dent Mater 29:e20-e28. doi:10.1016/j. dental.2012.11.007

15. Camilleri J, Sorrentino F, Damidot D (2013) Investigation of the hydration and bioactivity of radiopacified tricalcium silicate cement, Biodentine and MTA Angelus. Dent Mater 29:580-593. doi:10.1016/j.dental.2013.03.007

16. Raskin A, Eschrich G, Dejou J, About I (2012) In vitro microleakage of Biodentine as a dentin substitute compared to Fuji II LC in cervical lining restorations. J Adhes Dent 14:535542. doi:10.3290/j.jad.a25690

17. Koubi G, Colon P, Franquin JC, Hartmann A, Richard G, Faure MO, Lambert G (2013) Clinical evaluation of the performance and safety of a new dentine substitute, Biodentine, in the restoration of posterior teeth - a prospective study. Clin Oral Investig 17:243249. doi:10.1007/s00784-012-0701-9

18. Li X, De Munck J, Pongprueksa P, Van Landuyt K, Van Meerbeek B (2014) Chemical interaction of calcium-silicate cements with demineralized dentin. J Dent Res 93(Spec Iss B):Abstr. No. 14

19. Hashem D, Mannocci F, Patel S, Manoharan A, Brown JE, Watson TF, Banerjee A (2015) Clinical and radiographic assessment of the efficacy of calcium silicate indirect pulp capping: a randomized controlled clinical trial. J Dent Res 94:562-568. doi:10.1177/ 0022034515571415

20. Reyes-Carmona JF, Felippe MS, Felippe WT (2009) Biomineralization ability and interaction of mineral trioxide aggregate and white Portland cement with dentin in a phosphatecontaining fluid. J Endod 35:731-736. doi:10.1016/j.joen.2009. 02.011

21. Han L, Okiji T (2011) Uptake of calcium and silicon released from calcium silicate-based endodontic materials into root canal dentine. Int Endod J 44:1081-1087. doi:10.1111/j.1365-2591.2011.01924.x

22. Atmeh AR, Chong EZ, Richard G, Festy F, Watson TF (2012) Dentin-cement interfacial interaction: calcium silicates and polyalkenoates. J Dent Res 91:454-459. doi:10.1177/ 0022034512443068

23. Wang Y, Yao X (2010) Morphological/chemical imaging of demineralized dentin layer in its natural, wet state. Dent Mater 26:433-442. doi:10.1016/j.dental.2010.01.002

24. Guze K, Short M, Sonis S, Karimbux N, Chan J, Zeng H (2009) Parameters defining the potential applicability of Raman spectroscopy as a diagnostic tool for oral disease. J Biomed Opt 14:014016. doi:10.1117/1.3076195

25. Schulmerich MV, Cole JH, Kreider JM, Esmonde-White F, Dooley KA, Goldstein SA, Morris MD (2009) Transcutaneous Raman spectroscopy of murine bone in vivo. Appl Spectrosc 63:286-295

26. Friel JJ, Lyman CE (2006) X-ray mapping in electron-beam instruments. Microsc Microanal 12:2-25

27. Hoyer I, Gaengler P, Bimberg R (1984) In vivo remineralization of human enamel and dental calculus formation. J Dent Res 63:11361139

28. Baena JR, Lendl B (2004) Raman spectroscopy in chemical bioanalysis. Curr Opin Chem Biol 8:534-539

29. Endo K, Hashimoto M, Haraguchi K, Ohno H (2010) Crystal growth by restorative filling materials. Eur J Oral Sci 118:489-493

30. Bezerra AC, Novaes RC, Faber J, Frencken JE, Leal SC (2012) Ion concentration adjacent to glass-ionomer restorations in primary molars. Dent Mater 28:e259-e263. doi:10.1016/j.dental.2012.08.014 
31. Cosslett VE, Duncumb P (1956) Microanalysis by a flying-spot Xray method. Nature 177:1172-1173

32. Ayars EJ, Jahncke CL, Paesler MA, Hallen HD (2001) Fundamental differences between micro- and nano-Raman spectroscopy. J Microsc 202:142-147

33. De Munck J, Li X, Pongprueska P, Van Ende A, Van Meerbeek B (2013) Correlative micro-Raman/EPMA analysis of the calciumsilicate cement interface with dentin. J Dent Res 92(Spec Iss B): Abstr. No.191

34. Grüner D, Fäldt J, Jansson K, Shen Z (2011) Argon ion beam polishing: a preparation technique for evaluating the interface of osseointegrated implants with high resolution. Int $\mathrm{J}$ Oral Maxillofac Implants 26:547-552

35. Robson AJ, Grishin I, Young RJ, Sanchez AM, Kolosov OV, Hayne M (2013) High-accuracy analysis of nanoscale semiconductor layers using beam-exit ar-ion polishing and scanning probe microscopy. ACS Appl Mater Interfaces 5:3241-3245. doi:10. 1021/am400270w
36. Gandolfi MG, Van Landuyt K, Taddei P, Modena E, Van Meerbeek B, Prati C (2010) Environmental scanning electron microscopy connected with energy dispersive X-ray analysis and Raman techniques to study ProRoot mineral trioxide aggregate and calcium silicate cements in wet conditions and in real time. J Endod 36: 851-857. doi:10.1016/j.joen.2009.12.007

37. Prati C, Gandolfi MG (2015) Calcium silicate bioactive cements: biological perspectives and clinical applications. Dent Mater 31: 351-370. doi:10.1016/j.dental.2015.01.004

38. Qi YP, Li N, Niu LN, Primus CM, Ling JQ, Pashley DH, Tay FR (2012) Remineralization of artificial dentinal caries lesions by biomimetically modified mineral trioxide aggregate. Acta Biomater 8:836-842. doi:10.1016/j.actbio.2011.10.033

39. Gandolfi MG, Taddei P, Siboni F, Modena E, De Stefano ED, Prati C (2011) Biomimetic remineralization of human dentin using promising innovative calcium-silicate hybrid "smart" materials. Dent Mater 27:1055-1069. doi:10.1016/j.dental. 2011.07.007 